\title{
Internetwork and intranetwork communications during bursting dynamics: Applications to seizure prediction
}

\author{
S. Feldt ${ }^{1, *}$ H. Osterhage, ${ }^{2,3}$ F. Mormann, ${ }^{2,4}$ K. Lehnertz, ${ }^{2,3,5}$ and M. Żochowski ${ }^{1,6}$ \\ ${ }^{1}$ Department of Physics, University of Michigan, Ann Arbor, Michigan 48109, USA \\ ${ }^{2}$ Department of Epileptology, University of Bonn, Bonn, Germany \\ ${ }^{3}$ Helmholtz-Institute for Radiation and Nuclear Physics, University of Bonn, Bonn, Germany \\ ${ }^{4}$ California Institute of Technology, Division of Biology, 216-76, Pasadena, CA 91125, USA \\ ${ }^{5}$ Interdisciplinary Center for Complex Systems, University of Bonn, Bonn, Germany \\ ${ }^{6}$ Biophysics Research Division, University of Michigan, Ann Arbor, Michigan 48109, USA \\ (Received 9 March 2007; revised manuscript received 23 May 2007; published 20 August 2007)
}

\begin{abstract}
We use a simple dynamical model of two interacting networks of integrate-and-fire neurons to explain a seemingly paradoxical result observed in epileptic patients indicating that the level of phase synchrony declines below normal levels during the state preceding seizures (preictal state). We model the transition from the seizure free interval (interictal state) to the seizure (ictal state) as a slow increase in the mean depolarization of neurons in a network corresponding to the epileptic focus. We show that the transition from the interictal to preictal and then to the ictal state may be divided into separate dynamical regimes: the formation of slow oscillatory activity due to resonance between the two interacting networks observed during the interictal period, structureless activity during the preictal period when the two networks have different properties, and bursting dynamics driven by the network corresponding to the epileptic focus. Based on this result, we hypothesize that the beginning of the preictal period marks the beginning of the transition of the epileptic network from normal activity toward seizing.
\end{abstract}

DOI: 10.1103/PhysRevE.76.021920

PACS number(s): 87.18.Hf, 87.18.Sn, 05.45.Xt

\section{INTRODUCTION}

Epilepsy, one of the most common neurological disorders, is characterized by the sudden onset of recurrent seizures due to a hypersynchronous firing of populations of neurons. Due to the debilitating nature of seizures and the fact that approximately $1 \%$ of the world population suffers from epilepsy, much research has investigated the dynamics of the onset of seizures with the hopes of developing methods of seizure prediction [1]. One of the most common types of epilepsy is focal epilepsy in which seizures originate from a circumscribed region within the brain. Since only about $2 / 3$ of epilepsy patients will respond to medication, surgery to remove the focal region is another option for treatment [2]. In order to locate the focus, clinicians rely on information from electroencephalogram (EEG) recordings combined with various imaging techniques. The availability of EEG data from these patients has allowed researchers to study the dynamics of the EEG signal before and during a seizure in hopes of better understanding the seizure generating process with the ultimate goal of predicting seizures.

Through the analysis of EEG recordings, it has recently been shown that one can identify a preictal period before the onset of a seizure during which various properties of the EEG time series differ from those during interictal (activity between seizures) and ictal (seizure) periods [3]. Attempts have been made to characterize this preictal period using, among others, the largest Lyapunov exponent [4], correlation density [5], correlation dimension [6,7], and dynamical similarity measures $[8-10]$. Other recent approaches utilize mea-

\footnotetext{
*sarahfel@umich.edu
}

sures of phase synchrony which determines the degree of phase locking between two oscillatory signals. Although the concept of phase synchronization has long been known [11], it is only recently that it has been applied to nonlinear time series analysis [12] and biological data in the analysis of Parkinson patients [13], the cardiovascular and respiratory systems [14], the calcium oscillations of epileptic cultures of astrocytes [15,16], and in EEG recordings [17-20].

Measures of phase and lag synchronization show a rather unexpected effect: A significant decrease in synchronization between certain EEG channels during the preictal period. The patterning of channels that exhibit this drop is quite complicated and is not necessarily dependent upon spatial structure. It has been hypothesized that this is due to the fact that the spatial and functional structure of the brain do not overlap [19]. Specifically, structures that are far in terms of Euclidian distance may have strong functional links, while neighboring regions may be functionally independent. This leads one to believe that the drop in synchronization occurs in weakly connected, functionally different regions of the brain. Thus while the cause of this decrease is unknown, it has been hypothesized $[17,19]$ that the recordings are performed in separate regions of synchronized activity where one site has become involved in the synchronous activity associated with the epileptic focus and onset of the seizure, while the other site has yet to become enveloped in this activity.

Testing this hypothesis experimentally as well as understanding its dynamical underpinnings is difficult since the EEG records the activity from a population of neurons, and while EEG recordings give important information about neural activity, the recorded signal cannot be directly linked to the underlying dynamics of the brain. We thus turn to a mod- 
eling approach to gain further insight into the possible mechanisms for the increased synchrony observed during interictal periods as well as the drop in synchrony during the preictal period.

We study a computational model in which two coupled networks of integrate-and-fire neurons model separate EEG recording sites. We choose one network to be associated with the seizure generating region (epileptic focus) and slowly drive this pathological network into a bursting (seizing) state by increasing the excitability of the neurons within that network over time. This method of transition into a seizure is chosen to mimic a class of cellular mechanisms thought to lead to a seizure [21]. A model of this type allows for analysis of the levels of synchronization over the total population of the networks (similar to using intracranial EEG) as well as at the level of the individual neurons.

We observe changes within the collective dynamics of the pathological network as the neurons transition from the globally asynchronous firing state which we consider to represent normal neural dynamics into the bursting state of a seizure. If the collective dynamics of the networks share gross dynamical properties (i.e., the same excitability), the networks will enter a resonance state. This leads to an amplification of the intrinsic oscillatory rhythm and increased levels of locking between collective signals of the networks. However, as the network corresponding to the epileptic focus begins its transition into the seizing state (but before the network begins to burst), the networks stop resonating, resulting in an elimination of the oscillatory patterning and a subsequent drop in phase synchrony that marks the beginning of the preictal period. During this time, the neurons of the pathological network continue to fire asynchronously but begin to increasingly lock their frequencies. Once the pathological network reaches the bursting state, it begins to drive the other network into a bursting state and we again see the high levels of synchronization characteristic of the ictal period.

We thus postulate that the preictal period marks the beginning of the transition from normal neural dynamics into bursting dynamics, which is characterized by the steady increase and locking of neuronal frequencies that eventually leads to bursting. This transition in the "focal" network is accompanied by an initial lack of a similar transition in the "normal" network, which causes the divergence of intrinsic network properties and a drop in the phase synchrony between the two networks.

\section{METHODS}

\section{A. Model}

The system studied in the paper consists of two interconnected small-world networks (SWNs) of integrate-and-fire neurons. Each network consists of 225 neurons situated on a two-dimensional (2D) square lattice with a lattice constant of $a=1$ and periodic boundary conditions. Neurons are initially locally connected to neighbors within a radius of $k=2$. The connections are then rewired with a probability of $p=0.3$, consistent with the Watts-Strogatz small-world (SW) model [22]. The small-world architecture has been shown to pro- duce self-sustained activity [23], increase the network's ability to synchronize [24,25], and has previously been used for models of epileptic behavior [26-28].

We introduce connections between the two networks by selecting a fraction $f=0.5$ of the neurons in each network to send synaptic current to randomly chosen $m$ neurons in the other network. Unless stated otherwise, $m=15$. Connecting the networks in this manner causes the neurons in one network to receive, on average, seven random connections from the other network, representing the average activity of that network.

The dynamics of each neuron are governed by

$$
\tau_{m} \frac{d V_{i}}{d t}=-\alpha_{i} V_{i}(t)+\sum_{j \in \delta} J_{i j}(t)+B \sum_{k \in \gamma} J_{i k}(t)+\xi_{i}(t)+E,
$$

where $\tau_{m}=20 \mathrm{~ms}, \alpha_{i}$ is the leakage coefficient which is uniformly distributed in $[1,1.1], \delta$ represents the intranetwork connections, $\gamma$ represents the internetwork connections of the $i$ th neuron, and we sum over the incoming synaptic current $J$. $B$ is the coupling parameter between the networks. For the simulations, we used $B=0.4$ unless otherwise noted. The noise variable for each neuron $\xi_{i}(t)$ is uniformly distributed in $[0,0.5]$ and $E$ is the excitability parameter which is constant for each neuron in a given network, but is allowed to vary between the networks. The neural excitability determines the amount of synaptic input needed to cause the neuron to fire and acts as a control parameter between the asynchronous firing of neurons within a network and bursting behavior where the population of neurons fires collectively. We use this parameter to induce bursting in one network by slowly raising its value over time, bringing the network from a nonbursting state into a bursting state. The level of excitability at which the network transitions into the bursting state is referred to as the bursting threshold. We will denote the two networks as $N 1$ and $N 2$ with respective excitability parameters $E_{1}$ and $E_{2}$.

Equation (1) was integrated using Euler's method and a neuron was said to fire an action potential when the membrane potential $V$ reached a threshold value of 1 . At this point, the neuron emits a spike of synaptic current that is sent to the neurons to which it is connected. For this reason we use the term "spike" to refer to the firing of a neuron. The incoming synaptic current to the $i$ th neuron from the $j$ th neuron is given by

$$
J_{i j}(t)=A\left[\exp \left(-\frac{t-t_{j}}{\tau_{s}}\right)-\exp \left(-\frac{t-t_{j}}{\tau_{f}}\right)\right],
$$

where $t_{j}$ denotes the last time at which the $j$ th neuron spiked, $\tau_{s}=0.2 \mathrm{~ms}$ is the slow time constant, and $\tau_{f}=0.02 \mathrm{~ms}$ is the fast time constant. These two time constants determine the spike shape and are chosen to approximate a biological action potential [29]. The parameter $A=1.8$ sets the amplitude of the spike. After firing, the membrane potential is reset to 0 , and the neuron enters a refractory period of $8 \mathrm{~ms}$ under which it does not integrate incoming current. Moreover, when out of the refractory period, a neuron only integrates synaptic current if the total value is above a threshold level of 0.4. Each network has an intranetwork synaptic delay of 
$0.6 \mathrm{~ms}$ and unless stated otherwise, the internetwork delay is $0.8 \mathrm{~ms}$. These two constants define the spatial extent and the distance between the two networks.

For comparison with EEG recordings, we consider here the total current signal of each network which we define to be the sum of all synaptic currents of the neurons within the network at each point in time, as this tells us the collective activity of a population of neurons. We can then look at measures of phase and lag synchrony between the total current signals of our networks while varying their relative properties and compare the results to those obtained from EEG recordings of epileptic patients. Our model also allows us to monitor the firings of the individual neurons within the network and to study how the synchronization of the individual neurons leads to the observed signal. This allows for insight into the mechanisms behind the synchronization of the two networks on the neuronal level which is difficult to obtain from actual EEG recordings.

\section{B. Mean phase coherence}

We first examine the mean phase coherence in our system which is a measure of phase synchrony. Phase synchrony refers to the state where the phases of two oscillators become locked while their amplitudes remain uncorrelated [30]. This generally occurs in systems of weakly coupled, nonidentical oscillators. We consider the brain to be an example of such systems, as a first order approximation, since each neuron is different, and the total number of synapses a given neuron has is small compared to the total number of neurons in the brain. The general definition of phase locking in noisy oscillators is

$$
\Delta \phi_{1,2}=\left|k \phi_{1}-l \phi_{2}\right| \cong \text { const },
$$

where $\phi$ denotes the phase of the oscillators and $k$ and $l$ are integers (here we use $k=l=1$ ). The mean phase coherence examines the angular distribution of the difference in phase between two oscillators and is defined [17] as

$$
R=\left|\frac{1}{N} \sum_{j=0}^{N-1} e^{i \Delta \phi_{1,2}(j \Delta t)}\right|,
$$

where $N$ denotes the number of samples in a discrete time series and $1 / \Delta t$ is the sampling rate. This definition restricts $R \in[0,1]$ and phase locking occurs for $R=1$ while $R=0$ implies unsynchronized signals.

To calculate $R$ for our simulations, we used a moving window technique in accordance with [18] with $k=l=1$. The time series of the collective signal was divided into a series of windows composed of 4096 points or $819.2 \mathrm{~ms}$ with an overlap of $20 \%$. First, the data in each window were demeaned (the mean value of the signal was subtracted, eliminating any dc component of the signal), and a Hanning window was applied. We then used the Hilbert transform [31] to define the analytic signal and calculate the instantaneous phase of the signal.

The Hilbert transform of a signal $s(t)$ is given by

$$
\widetilde{s}(t)=\frac{1}{\pi} P \cdot V \cdot \int_{-\infty}^{\infty} \frac{s(\tau)}{t-\tau} d \tau
$$

(where P.V. denotes the Cauchy principal value) and the analytic signal is then defined as

$$
\zeta(t)=s(t)+i \widetilde{s}(t) .
$$

From this, we can uniquely define the instantaneous phase of our signal as

$$
\phi(t)=\arctan \left(\frac{\widetilde{s}(t)}{s(t)}\right) .
$$

Finally, $10 \%$ of the signal was discarded at both ends to minimize the edge effects caused by applying the Hilbert transform to a finite signal. The resulting phases were used to calculate the phase difference between signals and the mean phase coherence.

\section{Maximum linear cross correlation}

We also examine the lag synchronization of the total current signal. Lag synchronization refers to the case when the state variables of two signals are the same but offset by a constant time lag [32]. A measure of lag synchronization between two signals $s_{1,2}(t)$ at a time lag $\tau$ is the normalized cross correlation given by

$$
\hat{C}\left(s_{1}, s_{2}\right)(\tau)=\left|\frac{C\left(s_{1}, s_{2}\right)(\tau)}{\sqrt{C\left(s_{1}, s_{1}\right)(0) C\left(s_{2}, s_{2}\right)(0)}}\right|,
$$

where $C$ is the linear cross correlation function,

$$
C\left(s_{1}, s_{2}\right)(\tau)=\int_{-\infty}^{\infty} s_{1}(t+\tau) s_{2}(t) d t .
$$

In order to measure the lag synchronization of our system, we look at the maximum linear cross correlation [18] defined as

$$
C_{\max }=\max _{\tau}\left\{\hat{C}\left(s_{1}, s_{2}\right)(\tau)\right\} .
$$

As with the case of the mean phase coherence, $C_{\max } \in[0,1]$, and $C_{\max }=1$ implies complete lag synchronization while $C_{\max }=0$ for unsynchronized signals. We use the moving window technique described above to calculate $C_{\max }$ over each window.

\section{RESULTS}

We observe three different types of behavior in the total current signal of the modeled network as seen in Fig. 1. In an uncoupled system, the network will undergo random firing for low values of $E$ as seen in Fig. 1(a). As the value of $E$ is increased and the mean firing rates of the neurons increase, the network enters an oscillating stage where the total current signal undergoes oscillatory modulation as in Fig. 1(b). When $E$ is increased further, the network reaches the bursting threshold where the neurons begin to fire synchronously, and the network enters the bursting stage of Fig. 1(c). 


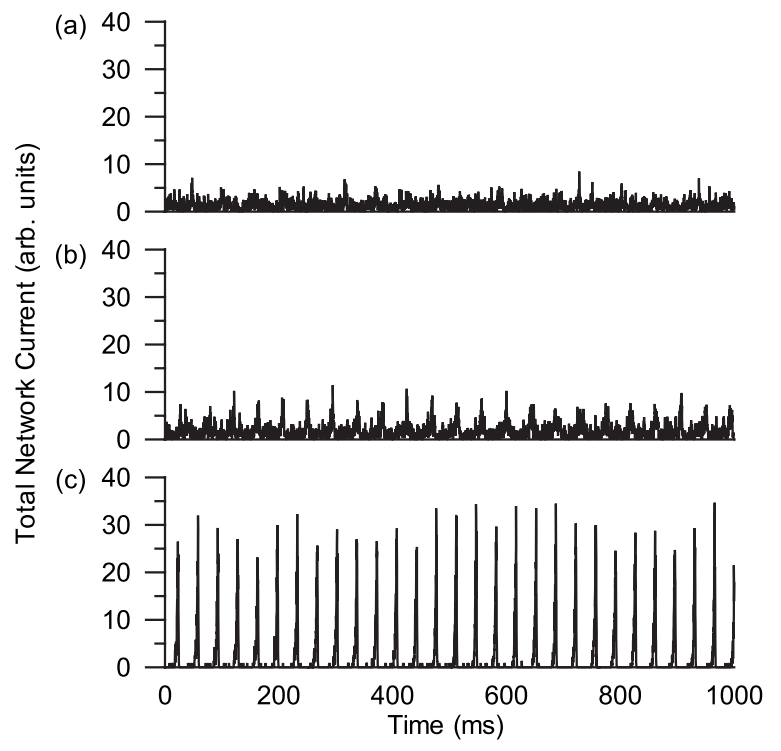

FIG. 1. Examples of the different types of behavior of the collective current trace of a single, uncoupled network. (a) Random firing behavior seen below the bursting threshold, $E=0.85$. (b) Fast oscillatory modulation just before the transition to bursting, $E$ $=0.95$. (c) Bursting behavior observed above the bursting threshold, $E=1.1$.

These results are consistent with the findings of [26], who studied single 1D SWNs and induced bursting behavior by adding additional long range connections to the network while holding the excitability of the neurons constant. Here, we hold the topology constant and induce the transition to bursting by increasing the excitability of the neurons to mimic the transition from interictal to ictal dynamics. It has been shown [33] that slices from the CA3 region of the hippocampus exhibit population bursts when the mean firing rate of the neurons within a driver site is increased above a threshold level. It has been hypothesized that bursting behavior within a neural network is thus the result of the increase in the mean firing rate of the neurons within a given region above some frequency threshold. This is precisely what we observe when increasing the excitability parameter due to the link between the excitability parameter and the firing rate of the neurons. In Fig. 2(a), we show this relationship between the average firing rate of five neurons and the excitability parameter. As we raise the excitability of the network, the neurons begin to fire more rapidly and the network enters a bursting state. As a result, there is also an increase in the mean current output by the network [Fig. 2(b)].

In a coupled network system, the excitability of $N 2$ is held constant at $E_{2}=0.8$, representing a local network which is not a part of the epileptic focus. Conversely, $N 1$ represents a local network that is part of the epileptic focus, and we step up the excitability of this network from $E_{1}=0.75$ to $E_{1}=1.1$ to obtain the transition to bursting, seizurelike dynamics. In Fig. 3 we show the mean phase coherence $R$ and the maximum linear cross correlation $C_{\max }$ plotted as a function of the difference in excitability between the networks, $\Delta E=E_{1}$ $-E_{2}$, averaged over 100 simulations. The average values of $R$ and $C_{\max }$ were calculated for each simulation by iterating for
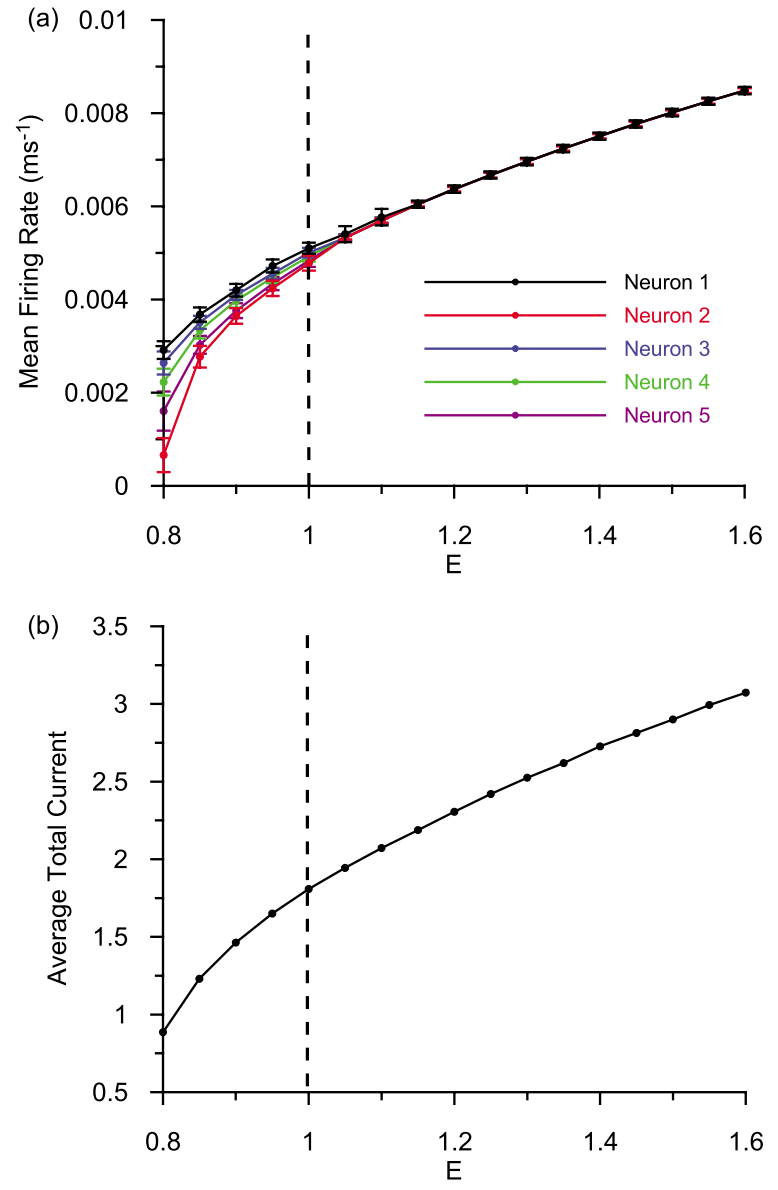

FIG. 2. (Color online) (a) Relationship between the excitability parameter of a single network and the average firing rate of five different neurons within the network. Increasing the excitability of the network causes the neurons to fire more rapidly and to synchronize. (b) The average total current in a single network as a function of the excitability. As the excitability is increased, the total synaptic current in the network will rise. The dotted line denotes the bursting threshold.

$10 \mathrm{~s}$ at each value of $\Delta E$ and disregarding a transient time of $4 \mathrm{~s}$.

We focus on the three dynamical regimes, when: (A) both networks are well below bursting threshold and have the same properties (same excitability, $\Delta E=0$ ), (B) both networks are below bursting threshold and $\Delta E \neq 0$, and (C) the neural excitability of $N 1$ is above the bursting threshold.

For low values of $E$, below the bursting threshold, when $E_{1}=E_{2}$, the total current signal of both networks remains asynchronous but exhibits slow oscillatory modulation as observed in a single network for relatively high network excitability. However, the oscillatory modulation observed here is due to the resonance drive of both networks through the internetwork coupling. We associate this regime with the interictal dynamics observed in the epileptic brain.

When the networks are below the bursting threshold but have significantly different properties such that $E_{1} \neq E_{2}$, even though the total input from $N 1$ to $N 2$ increases significantly as $E_{1}$ is increased, both networks still may exhibit asynchronous dynamics and, furthermore, the slow oscillatory modu- 


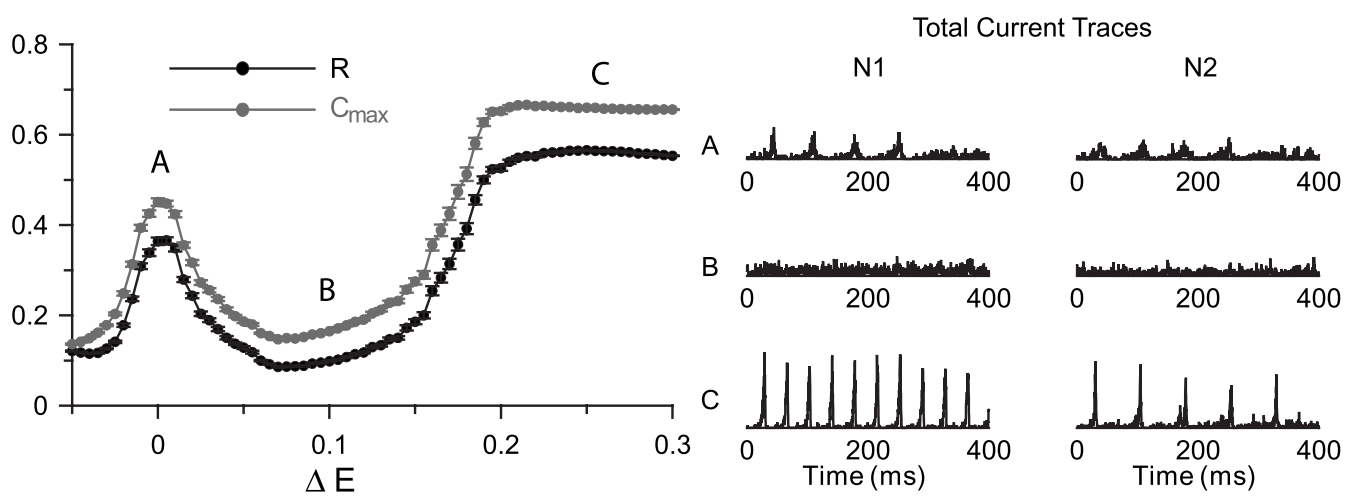

FIG. 3. Plots of the average phase coherence and maximum cross correlation coefficient as a function of the mismatch between the excitability parameters in the networks. Values were averaged over 100 simulations as described in the text. Sample current traces are shown for the different types of behavior seen during the resonance (A), random firing (B), and bursting regimes (C).

lation is abolished. We link this regime to the preictal state.

When $N 1$ is above the bursting threshold, the bursting of that network will induce bursting behavior in N2. This driving interaction that occurs during the bursting regime represents the spread of bursting behavior throughout different regions of the brain during a seizure (the ictal state).

We thus observe a typical resonance curve centered on $\Delta E=0$ which is where we see the oscillatory behavior of the networks that gives rise to the higher levels of synchronization. As $\Delta E$ is further increased, the frequency response of the two networks becomes mismatched, the slow oscillatory modulation is abolished, and there is a drop in the synchronization. The measures of synchronization rise again for large values of $\Delta E$, as this is the region where $E_{1}$ crosses the bursting threshold and the networks enter the bursting regime, with $N 1$ driving the bursting in $N 2$.
We then studied the role of the coupling and delay between the networks on the observable drop of phase synchrony during the preictal period. Figure 4(a) shows the average values of $R$ and $C_{\max }$ for $\Delta E=0$ (upper lines) and $\Delta E=0.1$ (lower lines) calculated over 20 simulations in which the coupling parameter $B$ was varied while the number of connections between the networks was held constant at $m=15$. As the coupling in the network increases, the level of synchrony at the resonance increases and for high levels of coupling, the synchrony during the parameter mismatch increases as well. We calculated the differences, $\Delta R=R(\Delta E$ $=0)-R(\Delta E=0.1)$ and $\Delta C_{\max }=C_{\max }(\Delta E=0)-C_{\max }(\Delta E=0.1)$ in Fig. 4(b). The peak in this curve is due to the interplay between the level of synchrony at resonance and the spread in the range of oscillations as the coupling is increased. We see that the difference between the synchrony at resonance

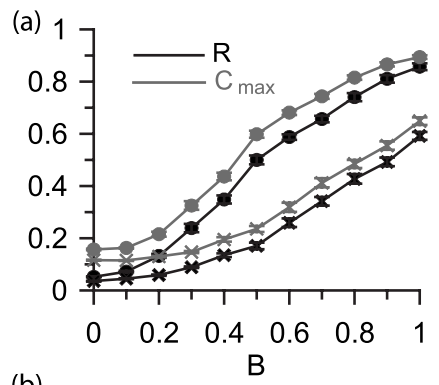

(b)

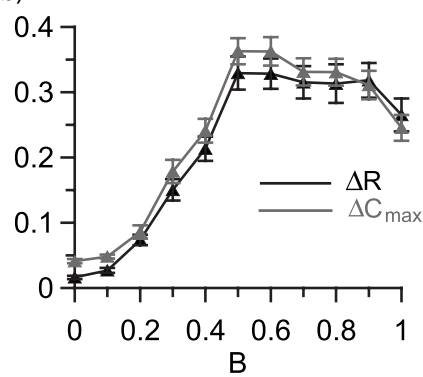

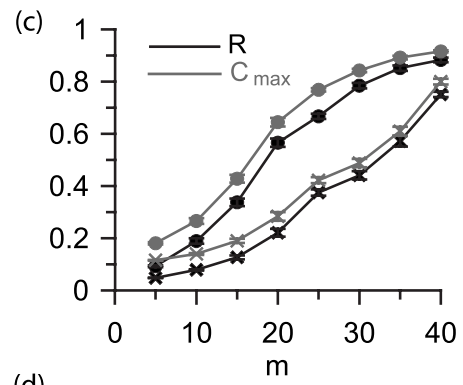

(d)

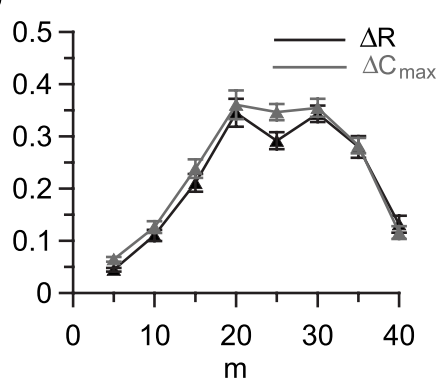

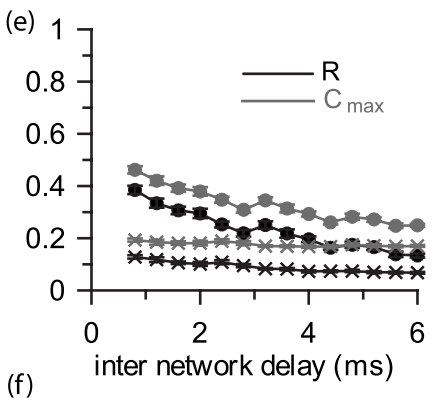

(f)

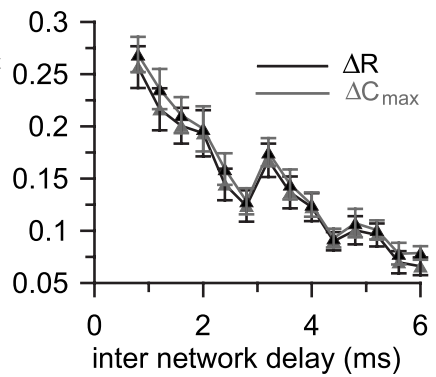

FIG. 4. (a, c, e) Average synchronization as a function of the coupling parameters during two regimes: $\Delta E=0-$ upper lines, and $\Delta E$ $=0.1$-lower lines. (b, d, f) Difference between the level of synchronization between the two regimes. (a) and (b) Calculated as a function $B$, with $m=15$. (c) and (d) Calculated as a function of the number of connections between networks $m$ with $B=0.4$. (e) and (f) Calculated as a function of internetwork synaptic delay with $B=0.4$ and $m=15$ with a constant intranetwork delay of $0.6 \mathrm{~ms}$. 

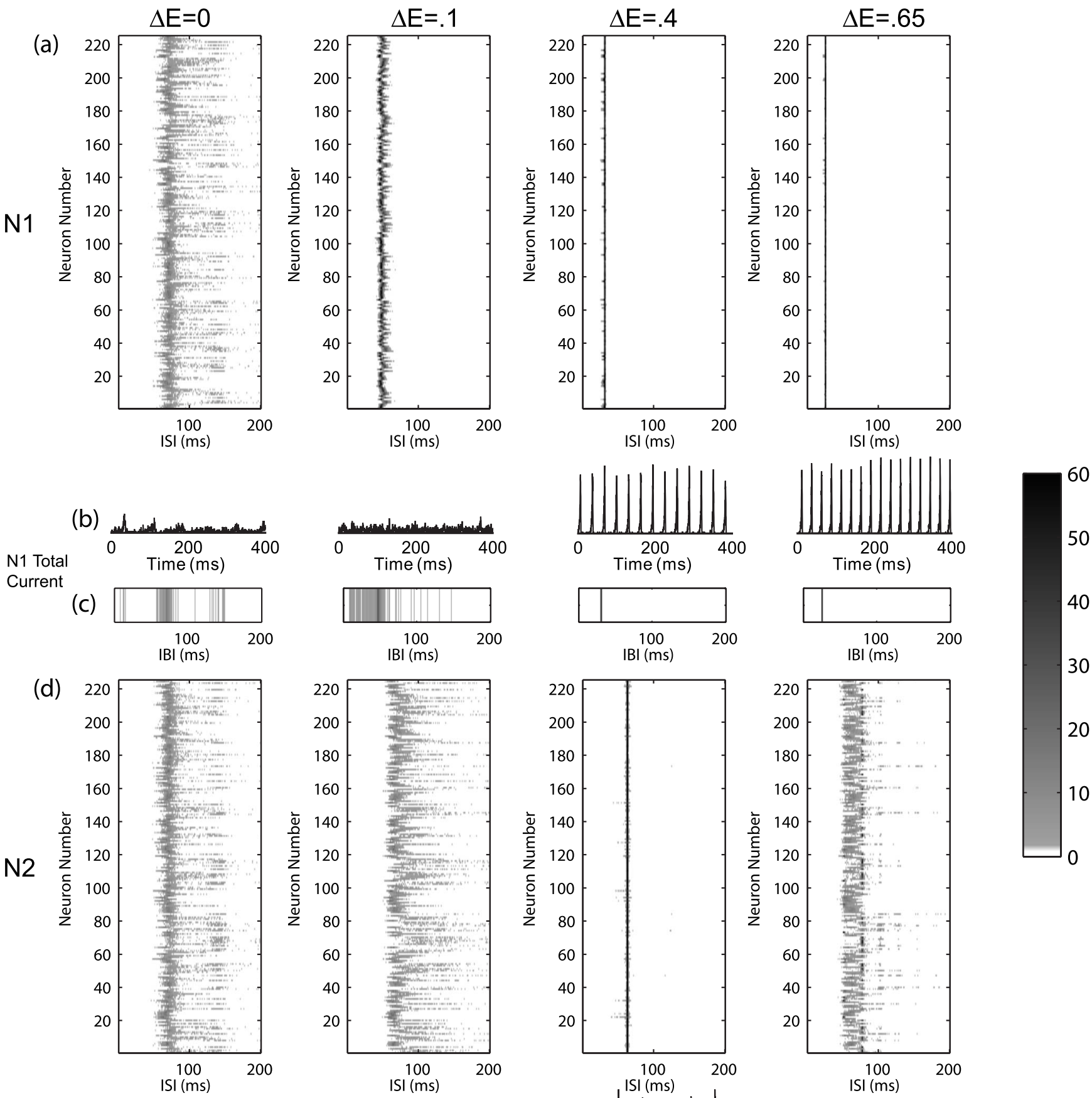

(e)
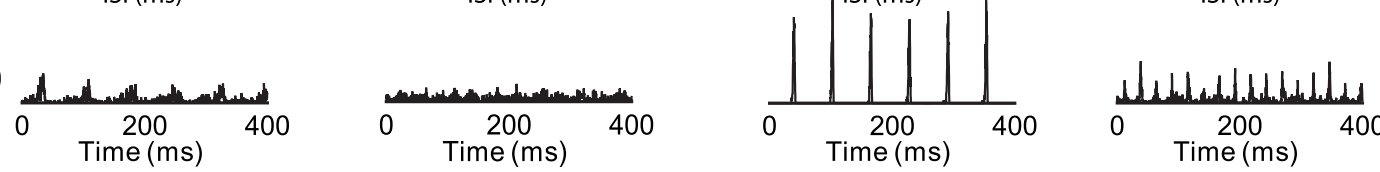

N2 Total

(f)
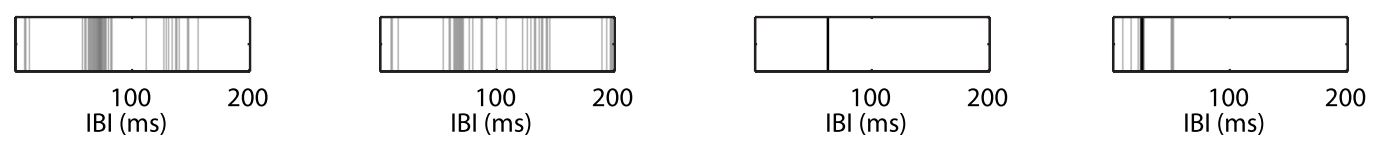

FIG. 5. Panels (a)-(c) show the analysis for N1, panels (d)-(f) show the analysis for N2. (a) and (d) Interspike interval (ISI) histograms for each neuron shown for four levels of excitability mismatch. (b) and (e) Samples of corresponding collective signals. (c) and (f) Interburst interval (IBI) histograms of the collective signal during the same intervals as in (a) and (c). Histograms were created by running a peak detection program on the collective signal to determine population spikes. Note that the ISI and IBI histograms do not necessarily correspond, indicating that the phase of the neuron plays a large role in the behavior of the network as a whole. 
and away from resonance remains high over a large range of coupling parameters. A similar effect was observed when we varied the number of connections $m$ between the networks and kept the internetwork coupling strength constant at $B$ $=0.4$ [Figs. 4(c) and 4(d)]. For large $m$, we see a decrease in the difference between synchrony levels. This is to be expected since as we add more internetwork connections, we are effectively losing the distinction of having two separate networks. We study the effects of internetwork delays on the observed phenomenon in Figs. 4(e) and 4(f). Here we hold the coupling parameters constant at $B=0.4$ and $m=15$. One can see that as the delay between the networks is increased, there is a decrease in the observed resonance peak. However, the behavior is observed over a significant range of delays indicating that the two interacting networks can be positioned relatively far apart.

In order to better understand the behavior of the networks in terms of the underlying neuronal dynamics, we examined the behavior of the individual neurons within each network. Figures 5(a) and 5(d) show histograms of the interspike intervals (ISIs) of the individual neurons within each network for four different values of $\Delta E$ in a system with $B=0.4$ and $m=15$. We also performed a peak detection to detect bursts in the total current signal of each network [Figs. 5(b) and 5 (e)] to create histograms of the interburst intervals (IBIs). The peak detection was done by smoothing the signal over a window of $1.8 \mathrm{~ms}$ and a burst was said to occur when the smoothed signal increased above a threshold value of 3 . These histograms of the networks' collective behavior are shown in Figs. 5(c) and 5(f). A bin size of $1 \mathrm{~ms}$ was used to create the histograms.

We first focus on the behavior of the network for values of $E_{1}$ below the bursting threshold. For the case of $\Delta E=0$, we see that the ISI histograms of neurons in each network as well as the IBIs of each network have a similar distribution. The networks are operating at the same frequency. We observe slow oscillatory modulation in the total activity of the networks and consequently increased phase synchronization between the networks. When $\Delta E=0.1$, we observe a different type of behavior. Although the individual neurons in $N 1$ are firing at an approximately locked rate leading to the narrow ISI distribution, the total current signal of the network shows a broad IBI distribution indicating that the neural activity remains asynchronous, and the total current signal of the network undergoes occasional random, low activity bursts. The neurons in $N 2$ show a virtually unchanged, wide distribution of ISI. The distribution of IBI is similar to that observed previously, but the slow oscillatory component in the total activity is no longer observed. Thus the asynchronous dynamics of the first network do not significantly alter the temporal dynamics of the second network. This is due to the fact that the neurons in $N 2$ receive a current input from $N 1$ which increases, but remains temporally unstructured.

When the value of $E_{1}$ is above the bursting threshold we observe different behavior. Both networks start to burst with evolving locking patterns. In the case of $\Delta E=0.4$, the ISI distributions of neurons in both $N 1$ and $N 2$ are highly peaked and the peaks correspond to those of the IBI distributions in the total activity of their respective networks, indicating that the neural populations within the networks are highly syn-

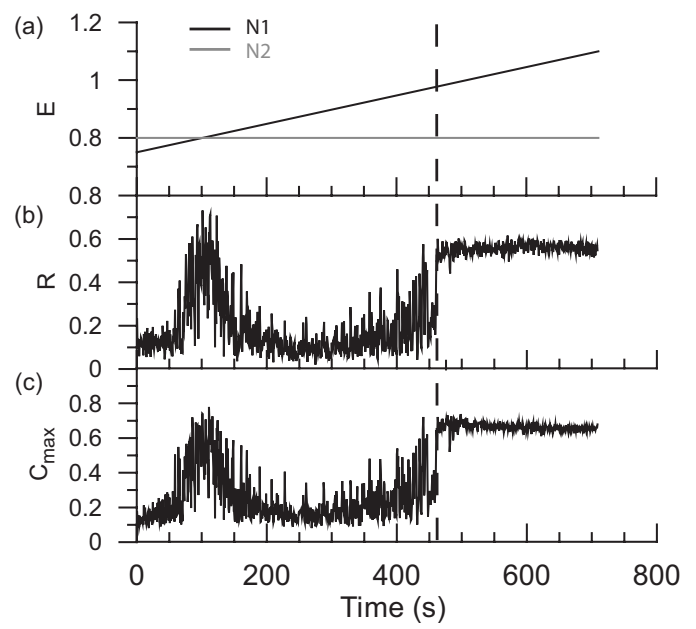

FIG. 6. Measures of synchronization. The vertical dashed line represents the bursting threshold. (a) Excitability parameters as a function of time. N1-black, N2-gray. (b) Phase coherence $R$ as a function of time. (c) Maximum cross correlation coefficient $C_{\max }$ as a function of time. High levels of synchronization occur during the region of parameter matching and during bursting behavior, while other regions exhibit low levels of synchronization.

chronized, and each network is now undergoing coherent bursting behavior. However, the value of ISI and IBI is different as the networks enter a 2:1 locking regime.

When $E_{1}$ is further increased $(\Delta E=0.65)$, we observe a transition to another type of behavior. The neurons in $N 1$ remain synchronized and the network bursts at a higher frequency due to the increase in the excitability. The neurons in $N 2$ become unsynchronized and fire roughly at multiples of the period of the neurons in N1. This leads to relatively weaker bursting, as a limited numbers of neurons fire in each cycle, but 1:1 locking can be observed.

Thus even though the frequency of spiking and the average magnitude of the mean-field signal of $N 1$ increases monotonically with changes of neuronal excitability, the slow oscillatory patterning in network activity observed when the networks have the same properties is initially abolished before the transition into bursting. This results in the drop of synchrony in the transitional period, before the onset of bursting. We thus hypothesize that the observed preictal drop in phase synchrony of the EEG is due to the abolition of resonant interaction between the two networks caused by changes in the frequency response of network that is associated with seizure generation. Therefore we postulate that the observed drop in synchrony is in fact an early signature of the pathological changes in the dynamics of the focus that eventually lead to seizure-type dynamics.

To demonstrate this transition from normal to pathological dynamics, we hold the excitability of $N 2$ constant at $E_{2}$ $=0.8$ and slowly increase the excitability of $N 1$ from $E_{1}$ $=0.75$ to $E_{1}=1.1$ as shown in Fig. 6 . The progression of the changes in synchrony, driven by incremental changes in excitability of $N 1$, mimics those observed in epileptic patients during transitions from interictal to preictal and preictal to ictal states.

In Fig. 7 we show, for comparison, the temporal course of the mean phase coherence $R$ estimated from EEG time series 


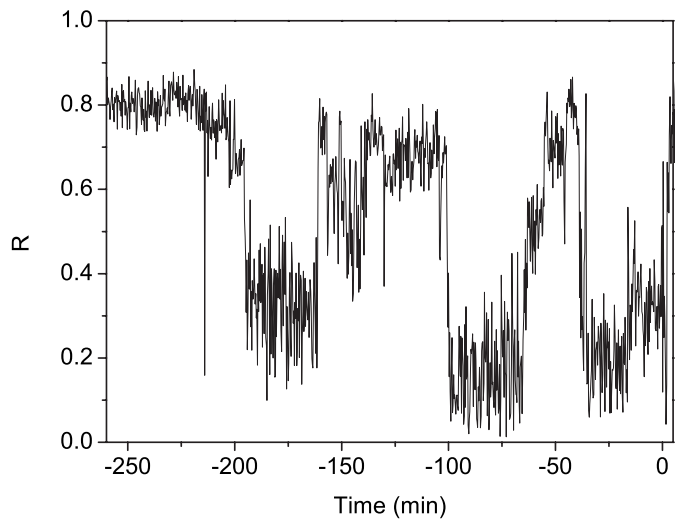

FIG. 7. Profile of the mean phase coherence $R$ for a pair of intrahippocampal EEG recordings from a patient suffering from mesial temporal lobe epilepsy. Seizure onset is at $t=0$.

that were recorded intrahippocampally from a patient suffering from mesial temporal lobe epilepsy during the phase preceding an epileptic seizure. EEG signals were sampled at $260 \mathrm{~Hz}$ using a 12 bit analog-to-digital converter and filtered within a frequency band of $0.5-85 \mathrm{~Hz} . R$ values were calculated (see Sec. II B) using a moving-window technique with nonoverlapping segments of $15.8 \mathrm{~s}$ corresponding to 4096 data points. In contrast to our model simulations, in the experimental setting we do not have access to the actual excitabilities of the network dynamics assessed by the respective EEG recordings. Nevertheless, we might speculate that the time course of $R$, in general, reflects fluctuations of the excitabilities of the network. Interestingly, during the time frame $-100-0$ min the course of $R$ and the fact that a seizure occurs is consistent with what we observed in our model when monotonically increasing the excitability of the "focal" network.

Thus here we define the preictal length to be the time it takes for the networks to transition from the resonance state into the bursting state and study the distribution of these lengths over multiple realizations of a given network (i.e., different instances of a network with the same global statistical properties). This can be seen in Fig. 8 for four runs on 12 different network realizations. The start of the preictal period was marked by the point at which the mean phase

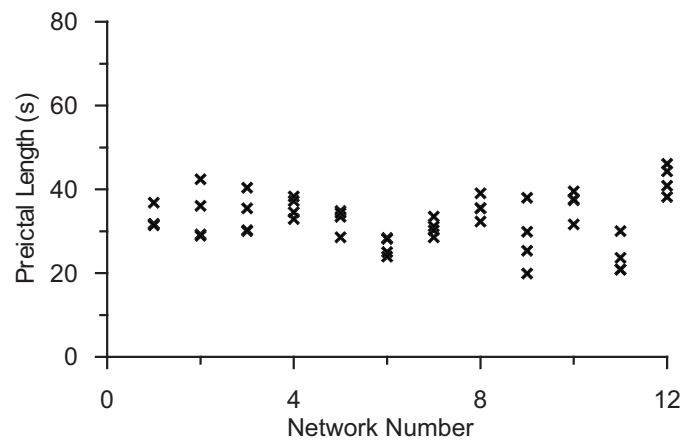

FIG. 8. Calculated preictal lengths for 12 different realizations of a network. Four simulations were run for each network realization and the preictal length was calculated as described in the text. coherence dropped below one standard deviation of its average value during the resonance state, and the end of the preictal period was defined to be a point at which the networks first entered the bursting state. We see that although each realization displays the transition from resonance to bursting, the time course varies for different realizations. This variance in preictal lengths between patients has also been observed experimentally [18].

\section{DISCUSSION AND CONCLUSIONS}

We have used a simple toy model of coupled networks to investigate the dynamical underpinnings of the drop in phase synchronization that is observed in epilepsy patients before a seizure. The observed regimes of high synchronization are the result of two types of interacting dynamics: a resonance interaction between the two networks when their properties are similar, and directional driving when the network associated with the focus drives the bursting in the other network.

We equate this resonance interaction between our networks to the normal dynamics of the brain during the interictal period and the directional driving to the propagation of the seizure during the ictal period. In between these two dynamical regions, we have an intermediate state which we equate to the preictal period where the dynamical properties of the interacting networks are mismatched, and the resonance interaction is abolished, while the directionally driven bursting is not yet present. While during both the resonance state and the driving state we see high levels of synchronization between the networks due to their similar dynamical properties, it is the mismatch of properties during the intermediate preictal state that leads to the observed decrease in the phase synchrony between the two networks.

We therefore compare the transition out of the resonance state and into the bursting state to the transition from normal neuronal dynamics to the pathological dynamics of a seizure. This implies that the observed drop in phase synchrony between certain EEG channels that defines the preictal period could be a result of the initial biological changes in the neurons associated with the focus and generation of the seizure that occur long before the system actually reaches the seizing state.

For this transition scenario to happen one has to assume that in the interictal period the focal and normal networks in the brain have similar gross dynamic properties so that they enter the oscillatory resonant state. There is experimental evidence from phase synchronization measures that interictal synchronization is high between electrodes placed within the same structure of the brain [17]. Such interactions are also observed between the focus and other brain regions during the interictal period [34]. Furthermore, it has become an accepted view in neuroscience that "the perpetual interactions among the multiple network oscillators keep cortical systems in a highly sensitive 'metastable' state and provide energyefficient synchronizing mechanisms via weak links" [35].

Although we have used a very simple model to explore a possible explanation for the underlying dynamics governing 
different areas of the brain before a seizure, our model shows the same behavior as observed in EEG recordings and has allowed us to make valuable insights at the neuronal level which cannot be done through the analysis of EEG recordings. We conclude that it is possible that the observed preictal period is a manifestation of initial biological neuronal changes that begin before the start of seizing behavior and encourage further experimental work to explore this hypothesis.

\section{ACKNOWLEDGMENTS}

S.F. was funded through the National Science Foundation. H.O. and K.L. acknowledge support from the Deutsche Forschungsgemeinschaft (Grant No. SFB-TR3 sub-project A2). F.M. acknowledges support from the European Community (6th Framework Programme, Marie Curie OIF 040445). M.Z. acknowledges support from NIBIB at NIH (Grant No. EB003583).
[1] F. Mormann, R. G. Andrzejak, C. E. Elger, and K. Lehnertz, Brain 130, 314 (2007).

[2] J. Engel, Surgical Treatment of Epilepsies (Raven Press, New York, 1987).

[3] F. Mormann, T. Kreuz, C. Rieke, R. G. Andrzejak, A. Kraskov, P. David, C. E. Elger, and K. Lehnertz, Clin. Neurophysiol. 116, 569 (2005).

[4] L. D. Iasemidis, J. C. Sackellares, H. P. Zaveri, and W. J. Williams, Brain Topogr. 2, 187 (1990).

[5] J. Martinerie, C. Adam, M. Le Van Quyen, M. Baulac, S. Clemenceau, B. Renault, and F. J. Varela, Nat. Med. 4, 1173 (1998).

[6] K. Lehnertz and C. E. Elger, Electroencephalogr. Clin. Neurophysiol. 95, 108 (1995).

[7] C. E. Elger and K. Lehnertz, Eur. J. Neurosci. 10, 786 (1998).

[8] M. Le Van Quyen, J. Martinerie, M. Baulac, and F. Varela, Neuroreport 10, 2149 (1999).

[9] M. Le Van Quyen, J. Martinerie, V. Navarro, P. Boon, M. D'Have, C. Adam, B. Renault, F. Varela, and M. Baulac, Lancet 357, 183 (2001).

[10] V. Navarro, J. Martinerie, M. Le Van Quyen, S. Clemenceau, C. Adam, M. Baulac, and F. Varela, Brain 125, 640 (2002).

[11] C. Huygens, Horologium Oscillatorum (Apud F. Muget, Paris, 1673).

[12] M. G. Rosenblum, A. S. Pikovsky, and J. Kurths, Phys. Rev. Lett. 76, 1804 (1996).

[13] P. Tass, M. G. Rosenblum, J. Weule, J. Kurths, A. Pikovsky, J. Volkmann, A. Schnitzler, and H. J. Freund, Phys. Rev. Lett. 81, 3291 (1998).

[14] C. Schafer, M. G. Rosenblum, J. Kurths, and H. H. Abel, Nature (London) 392, 239 (1998).

[15] G. Balazsi, A. Cornell-Bell, A. B. Neiman, and F. Moss, Phys. Rev. E 64, 041912 (2001).

[16] G. Balazsi, A. H. Cornell-Bell, and F. Moss, Chaos 13, 515 (2003).

[17] F. Mormann, K. Lehnertz, P. David, and C. E. Elger, Physica D 144, 358 (2000).

[18] F. Mormann, R. G. Andrzejak, T. Kreuz, C. Rieke, P. David, C. E. Elger, and K. Lehnertz, Phys. Rev. E 67, 021912 (2003).
[19] F. Mormann, T. Kreuz, R. G. Andrzejak, P. David, K. Lehnertz, and C. E. Elger, Epilepsy Res. 53, 173 (2003).

[20] M. Le Van Quyen, J. Soss, V. Navarro, R. Robertson, M. Chavez, M. Baulac, and J. Martinerie, Clin. Neurophysiol. 116, 559 (2005).

[21] J. A. Gorter, E. A. van Vliet, E. Aronica, and F. H. Lopes da Silva, Hippocampus 12, 311 (2002).

[22] D. J. Watts and S. H. Strogatz, Nature (London) 393, 440 (1998).

[23] A. Roxin, H. Riecke, and S. A. Solla, Phys. Rev. Lett. 92, 198101 (2004)

[24] T. Nishikawa, A. E. Motter, Y. C. Lai, and F. C. Hoppensteadt, Phys. Rev. Lett. 91, 014101 (2003).

[25] M. Barahona and L. M. Pecora, Phys. Rev. Lett. 89, 054101 (2002).

[26] T. I. Netoff, R. Clewley, S. Arno, T. Keck, and J. A. White, J. Neurosci. 24, 8075 (2004).

[27] B. Percha, R. Dzakpasu, M. Zochowski, and J. Parent, Phys. Rev. E 72, 031909 (2005).

[28] B. H. Singer, M. Derchansky, P. L. Carlen, and M. Zochowski, Phys. Rev. E 73, 021910 (2006).

[29] W. Gerstner and W. Kistler, Spiking Neuron Models: Single Neurons, Populations, Plasticity (Cambridge University Press, Cambridge, England, 2002).

[30] A. Pikovsky, M. Rosenblum, and J. Kurths, Synchronization: A Universal Concept in Nonlinear Sciences, Cambridge Nonlinear Science Series (University Press, Cambridge, England, 2001), Vol. 12.

[31] M. Rosenblum and J. Kurths, in Nonlinear Analysis of Physiological Data, edited by H. Kantz, J. Kurths, and G. MayerKress (Springer, Berlin, 1998), pp. 91-99.

[32] M. G. Rosenblum, A. S. Pikovsky, and J. Kurths, Phys. Rev. Lett. 78, 4193 (1997).

[33] L. M. de la Prida, G. Huberfeld, I. Cohen, and R. Miles, Neuron 49, 131 (2006).

[34] M. Le Van Quyen, J. Martinerie, C. Adam, and F. Varela, Physica D 127, 250 (1999).

[35] G. Buzsaki, Rhythms of the Brain (Oxford University Press, New York, 2006). 